\title{
Proceedings of the 2015 ISNR Conference: Keynotes, Invited, and Student Award Presentations
}

\author{
Selected Abstracts of Conference Presentations at the 2015 International Society for Neurofeedback \\ and Research (ISNR) 23rd Conference, Denver, Colorado, USA
}

Citation: International Society for Neurofeedback and Research. (2015). Proceedings of the 2015 ISNR Conference: Keynotes, Invited, and Student Award Presentations. NeuroRegulation, 2(4), 179-187. http://dx.doi.org/10.15540/nr.2.4.179

Copyright: ( 2015. ISNR. This is an Open Access article distributed under the terms of the Creative Commons Attribution License (CC-BY).

\section{KEYNOTE PRESENTATIONS}

\section{Developmental Trauma: Effects of Abuse and Neglect on CNS Development and a Possible Role for Neurofeedback to Reverse the \\ Damage \\ Bessel van der Kolk, MD}

Medical Director of The Trauma Center, Boston, Massachusetts, USA

Professor of Psychiatry at Boston University Medical School, Boston, Massachusetts, USA

Co-Director of the National Center for Child Traumatic Stress Complex Trauma Network, Boston, Massachusetts, USA

This keynote will discuss some of the wellestablished and direct affects of trauma on developing brains and specific brain functions responsible for attention, concentration, regulating emotions, and engaging in satisfying relationships. Dr. Kolk and colleagues are currently studying whether neurofeedback can reverse those brain changes. The importance of this pursuit is evident when considering that many traumatized children and adults continue to feel chronically on edge, scared, agitated, collapsed, and helpless, even after exposure to treatment and medications. To deal with this they often try to cope with alcohol or drugs. Medications may make life more manageable but they also affect motivation and curiosity, and rarely really lead to increased focus, relaxation, and engagement. Thus this lecture will review the way trauma impacts brain development and show the effects of neurofeedback.

\section{The Neuropsychopathology of Traumatic Brain Injury \\ Mark Gordon, MD}

Owner and Medical Director of Millennium-TBI, Encino, California, USA

In this presentation, Dr. Gordon will walk through some of the science that has helped his team provide treatment to patients where no improvement was thought possible. Some cases will be presented to illustrate the approach to diagnosis and treatment of Traumatic Brain Injury (TBI) for those who have failed to respond to traditional care. He will discuss at length the influence and relevance of neuropsychopharmacology, neuropsychobehavioral characteristics, and neuroplasticity to the diagnosis of TBI. Both genetic and epigenetic influences on neuroplasticity will be described, as will the details of hormonal mechanisms regulating reactive emotions from the limbic system, which influence intelligence and emotional presence. Additionally, the creation, importance, and delicate balance of neurosteroids with regard to behavioral reactions and mental wellbeing will be explained.

\section{Mindful Creativity}

Sayyed Mohsen Fatemi, PhD

Associate, Department of Psychology, Harvard University, Massachusetts, USA

This talk will explicate how Langerian mindfulness opens up a new horizon for exploring the novel possibility in the world of mind and body. In view of a distinction between meditation-based mindfulness and Langerian mindfulness, the talk will elucidate how Langerian mindfulness would give rise to developing new avenues of creativity with implications for health and well-being. In line with neuroplasticity and its focus on our power to influence the function and the structure of the brain, the talk will propound how Langerian mindfulness may provide alternative ways of looking at schema, cognition, thinking, and decision-making. 
Neuromodulation (TMS, tDCS, tRNS, tACS, neurofeedback): Working Mechanisms

Dirk DeRidder, MD, PhD

Founder and director of the $B R A I^{2} \mathrm{~N}$ (Brain Research Center for Advanced, Innovative and Interdisciplinary Neuromodulation), Antwerp, Belgium

The brain is an information-processing machine adjusting itself to the environment. Information processing is performed to reduce uncertainty, which is inherently present in a changing environment. Perception can be seen as Bayesian inference, where an intention-driven prediction is actively looked in the environment to update the prediction. The percept itself is an emerging property of network activation, processing the information at different oscillation frequencies for each subnetwork. Brain-related symptoms or diseases are associated with both activity, and even more importantly, connectivity changes. Using the brain's adaptive characteristics can be advantageous in retraining the brain by reshaping its networks. This is the purpose of neurostimulation and neurofeedback. Whereas neurostimulation interferes directly with activity and connectivity, neurofeedback or operant conditioning likely exerts its effect by interfering with the brain's Bayesian updating mechanisms.

A conceptual model of brain functioning can help to understand mechanisms involved in neurofeedback. This model relates delta oscillations to controlling basic homeostatic activity and a delta can be considered a carrier wave for higher oscillation frequencies, most likely beta activity. Theta is a memory-related carrier wave integrating beta3 and gamma activity by theta-beta and theta gamma nesting. Theta oscillations cover short- and longrange connections, whereas beta3 and gamma oscillations are more locally restricted in widely distributed areas. Alpha oscillations are thalamically driven and can be linked to attentional processes. Delta and beta1-2 is intermediate in its connectivity range. Theta/beta cross-frequency coupling possibly encodes memory-based predictions of future events/stimuli. In order to update the prediction, alpha is used as scanning frequency sampling the environment for salient information. The prediction error or change is encoded in gamma. Thus correct predictions about the environment will be encoded by beta activity, which therefore represents a status quo, whereas wrong predictions or insufficient input from the environment will be represented by beta/gamma cross-frequency coupling.
Thus neurofeedback attempts to modulate these oscillatory networks, thereby normalizing predictions or processing of prediction errors. This results in changing functional and effective connectivity as well as cross-frequency coupling.

\section{INVITED PRESENTATIONS}

\section{The Institute for Advanced Technology and Public Policy: Operation Headstrong Honorable Sam Blakeslee, $P h D^{1}$, and Lance lunker ${ }^{2}$ ${ }^{1}$ Former California State Senator; Founder of the Institute for Advanced Technology and Public Policy, California Polytechnic State University, San Luis Obispo, California, USA \\ ${ }^{2}$ Program Director, Institute for Advanced Technology and Public Policy, California Polytechnic State University, San Luis Obispo, California, USA; Retired U.S. Army Veteran}

The Institute for Advanced Technology is a think tank that seeks solutions to societal issues through technology, and then to shape public policy to make those solutions increasingly accessible. Currently the institute has four projects. One is Digital Democracy, an open government web-based tool. Two is Connect Academy, a tablet-based technology-in-the-classroom project geared towards lower income and challenged background schools seeking to incentivize parent participation in their child's learning. Three is CalWave, a sustainable wave energy project. The final project is Operation Headstrong, which utilizes emerging technology in neurofeedback therapy to improve the lives of veterans suffering from PTSD and TBI.

Lance lunker, an Iraq War veteran, will give an overview about Operation Headstrong. He will share his personal story about being wounded in Iraq and his struggles with PTSD and anxiety. His personal success story will demonstrate how neurofeedback therapy has helped him and he is now trying to help other veterans with neurofeedback therapy.

\section{STUDENT AWARD WINNERS - PLENARY PRESENTATIONS}

\section{Effects of Neurofeedback on Cognitive Profiles of College Students with ADHD Alycia Roberts, MA, and Scott Decker, PhD University of South Carolina, Columbia, South Carolina, USA}

Neurodevelopmental disorders such as ADHD represent a major national problem. There are increasing numbers of students in schools requiring special education services as a result of ADHD, and 
each of these students costs the U.S. education system approximately $\$ 5,000$ per year (Robb et al., 2011). There are additional societal costs associated with the disorder, and ADHD can be debilitating for individuals with the disorder and their families (i.e., Barkley \& Murphy, 2010; Ginsberg, Långström, Larsson, \& Lichtenstein, 2013). The most common treatments are stimulant medication and behavioral training (i.e., Pelham \& Fabiano, 2008), but recently neurofeedback (EEG biofeedback) has been receiving a lot of press. Both the American Academy of Pediatrics and the American Academy of Child and Adolescent Psychiatry have endorsed neurofeedback as a viable option for the treatment of ADHD (AAP, 2012; Lofthouse, Arnold, Hersch, Hurt, \& DeBeus, 2012).

Methods: The current study is a randomized controlled study investigating the effects of LORETA neurofeedback on a college population with ADHD. The study used a pre-test, multiple post-test design with delayed treatment to provide stronger evidence of its effectiveness. Both qEEG and behavioral data were collected to determine if there were changes in brain activity, and if these changes were evident on popular measures of cognitive ability (i.e., Woodcock-Johnson III) and attention (CPT-II).

Results: This presentation will provide group level analyses and case study reports of individuals from both conditions. Preliminary data are promising, suggesting the effectiveness of neurofeedback in a college population. The results of the full study will be presented.

Objectives: This $30-\mathrm{min}$ presentation will provide a brief overview of the literature regarding the use of neurofeedback with clinical populations, specifically individuals with ADHD. It will also provide a detailed account of the methodology used in the randomized control study described above, including the successes and limitations of the project. Finally, the results of the study will be discussed both regarding group level statistics, and matched control case studies to demonstrate the impact of the training protocol on the individual level, which is a primary area of interest for practitioners.

\section{References}

American Academy of Pediatrics. (AAP; 2012). SharpBrains.org. Retrieved from http://www.sharpbrains.com/blog/2012/10/05 /biofeedback-now-a-level-1-best-support-intervention-forattention-hyperactivity-behaviors/

Barkley, R. A., \& Murphy, K. R. (2010). Impairment in occupational functioning and adult ADHD: the predictive utility of executive function (EF) ratings versus EF tests. Archives of
Clinical Neuropsychology, 25(3), 157-173. http://dx.doi.org/10.1093/arclin/acq014

Ginsberg, Y., Långström, N., Larsson, H., \& Lichtenstein, P (2013). ADHD and criminality: could treatment benefit prisoners with ADHD who are at higher risk of reoffending? Expert Review of Neurotherapeutics, 13(4), 345-348. http://dx.doi.org/10.1586/ern.13.22

Lofthouse, N., Arnold, L. E., Hersch, S., Hurt, E., \& DeBeus, R. (2012). A review of neurofeedback treatment for pediatric ADHD. Journal of Attention Disorders, 16(5), 351-372. http://dx.doi.org/10.1177/1087054711427530

Pelham, W. E., \& Fabiano, G. A. (2008). Evidence-based psychosocial treatments for attention-deficit/hyperactivity disorder. Journal of Clinical Child and Adolescent Psychology, 37(1), 184-214. http://dx.doi.org/10.1080 $/ 15374410701818681$

Robb, J. A., Sibley, M. H., Pelham, W. E., Foster, E. M., Molina, B. S. G., Gnagy, E. M., \& Kuriyan, A. B. (2011). The Estimated Annual Cost of ADHD to the US Education System. School Mental Health, 3(3), 169-177. http://dx.doi.org/10.1007/s12310-011-9057-6

\section{Using Neurofeedback to Lower Anxiety} Symptoms Using Individualized qEEG Protocols: A Pilot Study

Stephanie Dreis, Angela Gouger, Michael Russo,

Edward Perez, and Mark S. Jones, DMin

The University of Texas at San Antonio, San Antonio, Texas, USA

Anxiety disorders affect approximately 40 million Americans ages 18 and over (NIMH, 2015). Various biofeedback modalities have been implemented by clinicians in the treatment of anxiety including electromyography (EMG), peripheral temperature, and electrodermal response (EDR) prior to the popularization of neurofeedback (Price \& Budzynski, 2009). Numerous studies illustrate the use of neurofeedback in correlation with symptom reduction in anxiety specifically but not limited to: frontal alpha asymmetry related anxiety (Kerson, Sherman, \& Kozlowski, 2009), psychiatric clients diagnosed with anxiety disorders (Cheon et al, 2013), performance anxiety in dancers (Singer, 2004), and anxiety symptoms related to posttraumatic stress disorder (PTSD; Walker, 2009). A study by Scheinost et al. (2013) showed participants with contamination anxiety having significantly more neural connectivity changes after undergoing neurofeedback treatment in comparison with the sham feedback group. Although many of the studies use set protocols on all patients, Hammond (2009) suggests the preferential nature of viewing the raw EEG when creating an individualized treatment protocol. This is due to other potentially confounding variables such as: co-morbid psychiatric conditions, medical issues, or effects from medication that the participants may possess. 
Prior research was closely examined in determination of each participant's protocol for this study. Although qualitative and small-scale quantitative studies show reduction in anxiety symptoms, large-scale studies or quantitative electroencephalography (qEEG)-driven protocols are non-existent. The creation of this pilot study intends to assess whether qEEG-guided amplitude neurofeedback is viable in symptom reduction of anxiety. Eight participants were prescreened to assess for anxiety. Two did not finish the treatment; therefore, their information was not used. Ages ranged from 15 to 52 (mean age 33.83). Half were women. Four of the participants identified as Hispanic/Latino and two identified as Caucasian. One participant had previous experience with neurofeedback, two participated in counseling during the study, five have had previous counseling experience, and three of the participants were currently taking medication. Pre- and postassessments to monitor behavior and symptoms were given to the participants. Assessments for adults included the Zung Self-Rating Anxiety Scale and the Adult Behavior Checklist (ABCL). Assessments for minors and their guardians included Screen for Child Anxiety Related Disorders (SCARED) and the Youth Behavior Checklist (YABCL). A qEEG was used to determine protocols for each participant. Participants were to receive 30min neurofeedback treatment sessions twice a week totaling 17 sessions. The range of attended session was 12-16, mean session attendance was 13.67.

Results will be known May 1st, 2015. Results will include the outcomes of pre- and post-assessments and qEEGs. An interesting finding includes having a participant with previous neurofeedback treatment use visualization techniques to produce anxiety and then use neurofeedback to help reduce anxiety. The current study has several limitations, such as a small sample size and no control group. Corrective suggestions are mentioned for future studies.

\section{References}

Cheon, E.-J., Koo, B.-H., Seo, W.-S., Lee, J.-Y., Choi, J.-H., \& Song, S.-H. (2015). Effects of neurofeedback on adult patients with psychiatric disorders in a naturalistic setting. Applied Psychophysiology and Biofeedback, 40(1), 17-24. http://dx.doi.org/10.1007/s10484-015-9269-x

Hammond, D. C. (2010). The Need for Individualization in Neurofeedback: Heterogeneity in QEEG Patterns Associated with Diagnoses and Symptoms. Applied Psychophysiology and Biofeedback, 35(1), 31-36. http://dx.doi.org /10.1007/s10484-009-9106-1

Hammond, D. C., Walker, J., Hoffman, D., Lubar, J. F., Trudeau, D., Gurnee, R., \& Horvat, J. (2004). Standards for the use of quantitative electroencephalography (qEEG) in neurofeedback: A position paper of the International Society for Neuronal Regulation. Journal of Neurotherapy, 8(1), 5-27. http://dx.doi.org/10.1300/J184v08n01_02

Kerson, C., Sherman, R. A., \& Kozlowski, G. P. (2009). Alpha Suppression and Symmetry Training for Generalized Anxiety Symptoms. Journal of Neurotherapy, 13(3), 146-155. http://dx.doi.org/10.1080/10874200903107405

National Institute of Mental Health. (NIMH; 2015). What are anxiety disorders? Retrieved from http://www.nimh.nih.gov/health/topics/anxietydisorders/index.shtml

Price, J., \& Budzynski T. (2009). Anxiety, EEG patterns, and neurofeedback. In T. H. Budzynski, H. K. Budzynski, J. R. Evans, \& A. Abarbanel (2nd Eds.), Introduction to Quantitative EEG and Neurofeedback: Advanced Theory and Applications (pp. 453-472). Burlington, MA: Elsivier, Inc. Retrieved from http://www.eblib.com

Scheinost, D., Stoica, T., Saksa, J., Papademetris, X., Constable, R. T., Pittenger, C., \& Hampson, M. (2013). Orbitofrontal cortex neurofeedback produces lasting changes in contamination anxiety and resting-state connectivity. Translational Psychiatry, 3(4), e250. http://dx.doi.org /10.1038/tp.2013.24

Singer, K. (2004). The effect of neurofeedback on performance anxiety in dancers. Journal of Dance Medicine and Science, $8(3), 78-81$.

Walker, J. E. (2009). Anxiety associated with post traumatic stress disorder-the role of quantitative electroencephalograph in diagnosis and in guiding neurofeedback training to remediate the anxiety. Biofeedback, 37(2), 67-70. http://dx.doi.org/10.5298/10815937-37.2.67

\section{STUDENT AWARD WINNERS - POSTER PRESENTATIONS}

\author{
Affective Virtual Reality Exposure with \\ Physiological Monitoring: Application for \\ Emotional Reactivity Testing in Autism \\ Yi Li, MA, Estate (Tato) Sokhadze, PhD, and Adel S. \\ Elmaghraby, PhD \\ University of Louisville, Louisville, Kentucky, USA
}

Background: Children with autism spectrum disorder (ASD) typically suffer from impairment in social skills, emotion recognition, and expression, and are at high risk of anxiety. They prefer to stay in a more protective and controllable environment. Virtual Reality (VR) is proven to be effective in a wide range of treatment sessions, such as exposure for patients with addictive disorders or social anxiety disorders (Meehan, Razzaque, Insko, Whitton, \& Brooks, 2005). However, it is very likely that the "standard" emotional response to the same VR scenarios between children on ASD and typical developed people would be different. Therefore, it is a reasonable approach to measure the differences in physiological responses during VR environment immersion, in order to design VR-based training or therapy sessions best suitable for ASD. 
Objectives: The study is based on using affective VR as a tool to induce emotional responses and measure autonomic nervous system (ANS) activity of children on ASD (Casanova et al., 2014) and in a group of typically developing control subjects. The main aim of this study is to characterize differences in autonomic reactivity between these two groups and select more usable measures for functional reactivity assessment, useful for social skills training targets.

Methods: Three affective VR scenarios are designed and chosen to evoke physiological responses during exposure to emotionally positive, negative, and neutral context. The hardware to provide VR is an Oculus Rift Development Kit 2, and the software platform is Unity 4.6 Pro with $\mathrm{C \#}$ as developing language. Autonomic nervous system variables are measured and recorded online by sensors attached to subjects during exposure the VR scenes. The autonomic dependent variables (Boucsein, 2012) include heart rate (HR), heart rate variability (HRV) measures (LF and HF, LF/HF ratio), skin conductance response (SCR), number of nonspecific SCR (NS.SCR) and respiration rate (RSP), along with several derived parameters. The system allowed monitoring the scenarios to synchronize it with autonomic recording using specific markers of events. Each scenario takes around $5 \mathrm{~min}$ for subjects to explore; one session requires exploration of all three scenes, which takes up to $20 \mathrm{~min}$ in total, including configuration, VR and sensors mount time. Recorded physiological data was analyzed using synchronized events and triggers from VR scenes for each emotional script. The subjects consisted of a group of five children with ASD and 10 typical controls.

Results and Conclusions: Preliminary results comparing skin conductance responses demonstrate a difference between ASD group and the control group. In particular, the frequency of NS.SCR to negative VR scenarios was significantly different between groups, with ASD group having higher, less differentiated NS.SCR across all conditions. SCR showed significant Emotion (neutral, negative) $X$ Group (ASD, controls) interaction. Heart rate responses and HRV measures showed group differences across emotional scripts. Application of VR with emotionally laden scripts in persons with ASD allows us to compare their ANS responses for better understanding of specifics of their emotional responsiveness, and use it for affective reactivity diagnostic and therapeutic purposes, including social skills training and biofeedback.

\section{References}

Boucsein, W. (2012). Electrodermal Activity (2nd ed.). New York: Springer.

Casanova, M. F., Hensley, M. K., Sokhadze, E. M., El-Baz, A. S., Wang, Y., Li, X., \& Sears, L. (2014). Effects of weekly lowfrequency rTMS on autonomic measures in children with autism spectrum disorder. Frontiers in Human Neuroscience, 8, 851. http://dx.doi.org/10.3389/fnhum.2014.00851

Meehan, M., Razzaque, S., Insko, B., Whitton, M., \& Brooks, F. P. (2005). Review of four studies on the use of physiological reaction as a measure of presence in stressful virtual environments. Applied Psychophysiology and Biofeedback, 30(3), 239-258. http://dx.doi.org/10.1007/s10484-005-6381-3

\section{EEG Coherence of Mu Rhythm in Successful and Unsuccessful Golf Putting Performance in Skilled Golfers \\ Kuo Pin Wang, MA ${ }^{1}$, Tai Ting Chen, $P h D c^{1}$, Jen Ying Su, $\mathrm{MA}^{2}$, Ming Yang Cheng, PhDc ${ }^{3}$, Chung Ju Huang, $\mathrm{PhD}^{2}$, Chen Hoa, $M A^{4}$, and Tsung Min Hung, $P h D^{1}$ \\ ${ }^{1}$ National Taiwan Normal University, Taipei, Taiwan \\ ${ }^{2}$ University of Taipei, Taipei, Taiwan \\ ${ }^{3}$ Cognitive Interaction Technology - Center of Excellence \\ (CITEC), Bielefeld University, Bielefeld, Germany \\ ${ }^{4}$ Chinese Culture University, Taipei, Taiwan}

In the sport area, golf putting is a motor skill that requires precise motor planning and a high level of concentration. Leocani (1997) demonstrated that the frontal and sensorimotor cortices are areas related to the planning and control of the motor skill. Previous study suggested that coherence analysis is a useful tool for understanding the functional connectivity between different cortical areas (Babiloni et al., 2011). Based on the findings of reduced mu power during the better motor preparation at premotor and sensorimotor cortices (Arnstein, Cui, Keysers, Maurits, \& Gazzola, 2011; Sabate, Llanos, Enriquez, \& Rodriguez, 2012), this study aimed to further investigate whether difference in mu coherence would be observed between successful and unsuccessful performance in precision motor control skill. In this study we recruited 35 skilled golfers. None of the participants had neurologic disease. All participants agreed with the experimental procedure and filled out the informed consent. According to this percentage, participants had to increase or decrease their putting distance in the next warm-up series. This process was repeated until the participant's distance of $50 \%$ accuracy was determined. The participants performed a total of 40 putts in four separate blocks (interblocks interval of about $1 \mathrm{~min}$ ) at an artificial golf green while Electroencephalography (EEG) were recorded and mu coherence (Fz-C3, Fz-C4, Fz-P3, Fz-P4, Fz-O1, Fz-O2, Fz-T3, Fz-T4) was derived. 2 (performance: successful performance, 
unsuccessful performance) $\times 2$ (time: T2 $=-2 \mathrm{~s} \sim$ $-1 \mathrm{~s}, \mathrm{~T} 1=-1 \mathrm{~s} \sim 0 \mathrm{~s}) \times 8$ (coherence sites: Fz-C3, FzC4, Fz-P3, Fz-P4, Fz-O1, Fz-O2, Fz-T3, Fz-T4) ANOVA revealed a significant interaction effect between performance and coherence sites. Post hoc simple main effect analysis indicated that the mu coherence for successful putting performance at FZ-C4 was smaller than that of unsuccessful performance. The results suggested that decreasing communication between motor planning and motor control of the left arm was related to better golf putting performance.

\section{References}

Arnstein, D., Cui, F., Keysers, C., Maurits, N. M., \& Gazzola, V. (2011). $\mu$-Suppression during Action Observation and Execution Correlates with BOLD in Dorsal Premotor, Inferior Parietal, and SI Cortices. Journal of Neuroscience, 31(40), 14243-14249. http://dx.doi.org/10.1523/Jneurosci.096311.2011

Babiloni, C., Infarinato, F., Marzano, N., lacoboni, M., Dassù, F., Soricelli, A., ... Del Percio, C. (2011). Intra-hemispheric functional coupling of alpha rhythms is related to golfer's performance: a coherence EEG study. International Journal of Psychophysiology, 82(3), 260-268. http://dx.doi.org /10.1016/j.ijpsycho.2011.09.008

Sabate, M., Llanos, C., Enriquez, E., \& Rodriguez, M. (2012). Mu rhythm, visual processing and motor control. Clinical Neurophysiology, 123(3), 550-557. http://dx.doi.org /10.1016/j.clinph.2011.07.034

Interaction Between EEG Control Training and EEG State Discrimination Training

Kelli N. Dunn ${ }^{1}$, Victor L. Pigg, Thomas F. Collura, $P h D^{2}$, and Jon Frederick, $P h D^{1}$

${ }^{1}$ Middle Tennessee State University, Murfreesboro, Tennessee, USA

${ }^{2}$ BrainMaster Technologies Inc., Bedford, Ohio, USA

It is commonly argued that the mechanism of action of biofeedback involves increasing awareness of subjective correlates of one's physiological state (Frederick, 2007, 2012). However, the relationship between awareness and control of physiological states is largely unexplored. Kamiya (1968) reported that those trained in alpha discrimination later showed superior performance in an alpha production task, but this finding was never replicated. Therefore, the present study seeks to determine whether subjects given standard neurofeedback (or "control") training will do better on EEG state discrimination (or "awareness") training, or vice versa. In a preliminary investigation, six subjects were given 4-7 sessions (median 7) of the following: (1) $5 \mathrm{~min}$ in which high alpha amplitude was rewarded; (2) 5 min during which low alpha was rewarded; (3) a repeat of (1); (4) a repeat of (2); followed by (5) 40 trials of alpha state discrimination training. Four subjects achieved a total of five criterion sessions (binomial $p<.05$ ) in the discrimination task. These five successful sessions occurred on days when the participant also succeeded in maximizing the amplitude difference between the high and low alpha reward conditions (average rank 2 out of 6). However, this apparent relationship was confounded by a strong effect of time on both variables. The mean amplitude difference between the high and low alpha reward conditions showed a positive learning curve, with session number explaining $r^{2}=49 \%$ of the variance. Similarly, performance in the discrimination task increased over time with session number explaining $r^{2}=46 \%$ of the variance. All five criterion sessions occurred in the fifth or later session. Shorter times between sessions appeared to improve discrimination performance. The five criterion sessions had a mean 4.4 days since the previous session, compared to 8.2 for the remaining sessions (previously seen in Frederick, 2012). A similar effect was not seen for the control task. A larger sample size is needed to evaluate the significance of these findings. Future studies will compare the success of learning between these two tasks alone vs. combined to examine whether there are synergistic effects.

\section{References}

Frederick, J. A. (2012). Psychophysics of EEG alpha state discrimination. Consciousness and Cognition, 21(3), 13451354. http://dx.doi.org/10.1016/j.concog.2012.06.009

Frederick, J. A. (2007, October). The role of mind-body medicine in the mind-body problem. NeuroConnections Newsletter, 15, 32-33

Kamiya, J. (1968, April). Conscious control of brain waves. Psychology Today, 1, 57-60.

\section{Exploring the Effects of Neurofeedback Training on Subjective and Objective \\ Performance in Elite Athletes \\ Adam O'Neil, MA', Max Sutton-Smolin ${ }^{2}$, Leslie Sherlin, $P h D^{3}$, Sarah Wyckoff, $P h D^{3}$, Markus Rogan, $M A^{2}$ \\ ${ }^{1}$ DISC Sports \& Spine Center, Marina Del Rey, California, USA \\ ${ }^{2}$ UCLA Anxiety Disorders Research Center, West Hollywood, California, USA \\ ${ }^{3}$ SenseLabs, Mesa, Arizona, USA}

Neurofeedback (Reinecke et al., 2011) training programs are increasingly used as strategies for enhancing athletic performance, due to their focus on increasing self-awareness and self-regulation (Faridnia, Shojaei, \& Rahimi, 2012). However, there is a need for more randomized controlled studies of the efficacy of these training programs using both subjective and objective performance measures. 
This randomized controlled trial was designed to study the effects of a neurofeedback training program on neurocognitive (EEG) and subjective self-report measures in an elite athlete population (university-level/Division I). The sample of elite athletes for this study was comprised of universitylevel NCAA Division I athletes from several different sports. The neurofeedback training protocol involves $10 \mathrm{hr}$ of Alpha enhancement training at occipital and parietal lobe sites. We hypothesize that participants receiving Alpha training will show demonstrable changes in EEG profile. During Phase I of data collection, researchers collected preand post-training data on 17 participants (eight in the neurofeedback group, nine in the control group). Data collection is still ongoing, but preliminary data suggest there could be significant findings that have not reached statistical significance due to lack of power. To further investigate this, the researchers are analyzing the neurofeedback and control groups' pre-post data to determine (a) whether greater neuro-electric change occurred in the neruofeedback treatment group and (b) whether these objective changes are correlated with selfreport performance measures. Limitations and future directions, including a description of Phase II of the data collection process, will be presented.

\section{References}

Aherne, C., Moran, A. P., \& Lonsdale, C. (2011). The effect of mindfulness training on athletes' flow: An initial investigation. Sport Psychologist, 25(2), 177-189.

Bakhshayesh, A. R., Hänsch, S., Wyschkon, A., Rezai, M. J., \& Esser, G. (2011). Neurofeedback in ADHD: a single-blind randomized controlled trial. European Child and Adolescent Psychiatry, 20(9), 481-491. http://dx.doi.org/10.1007/s00787011-0208-y

Baer, R. A., Smith, G. T., Hopkins, J., Krietemeyer, J., \& Toney, L. (2006). Using self-report assessment methods to explore facets of mindfulness. Assessment, 13(1), 27-45. http://dx.doi.org/10.1177/1073191105283504

Baer, R. A., Smith, G. T., Lykins, E., Button, D., Krietemeyer, J., Sauer, S., ... Williams, J. M. G. (2008). Construct validity of the five facet mindfulness questionnaire in meditating and nonmeditating samples. Assessment, 15(3), 329-342. http://dx.doi.org/10.1177/1073191107313003

Beauchamp, M. K., Harvey, R. H., \& Beauchamp, P. H. (2012). An integrated biofeedback and psychological skills training program for Canada's Olympic short-track speedskating team. Journal of Clinical Sport Psychology, 6(1), 67-84.

Bernier, M., Thienot, E., Codron, R., \& Fournier, J. F. (2009). Mindfulness and acceptance approaches in sport performance. Journal of Clinical Sports Psychology, 4, 320333.

Bishop, S. R., Lau, M., Shapiro, S., Carlson, L., Anderson, N. D., Carmody, J., ... Devins, G. (2004). Mindfulness: A proposed operational definition. Clinical Psychology: Science and Practice, 11(3), 230-241. http://dx.doi.org/10.1093 /clipsy.bph077

Breteler, M. H. M., Arns, M., Peters, S., Giepmans, I., \& Verhoeven, L. (2010). Improvements in spelling after QEEGbased neurofeedback in dyslexia: a randomized controlled treatment study. Applied Psychophysiology and Biofeedback, 35(1), 5-11. http://dx.doi.org/10.1007/s10484-009-9105-2

Brewer, J. (2014). Mindfulness in the military. American Journal of Psychiatry, 171(8), 803-806. http://dx.doi.org/10.1176 lappi.ajp.2014.14040501

Davidson, R. J. (2010). Empirical Explorations of Mindfulness: Conceptual and Methodological Conundrums. Emotion, 10(1), 8-11. http://dx.doi.org/10.1037/a0018480

Davis, P. A., \& Sime, W. E. (2005). Toward a psychophysiology of performance: sport psychology principles dealing with anxiety. International Journal of Stress Management, 12(4), 363-378. http://dx.doi.org/10.1037/1072-5245.12.4.363

Duckworth, A. L., \& Quinn, P. D. (2009). Development and Validation of the Short Grit Scale (Grit-S). Journal of Personality Assessment, 91(2), 166-174. http://dx.doi.org/10.1080/00223890802634290

Duric, N. S., Assmus, J., Gundersen, D., \& Elgen, I. B. (2012). Neurofeedback for the treatment of children and adolescents with ADHD: a randomized and controlled clinical trial using parental reports. BMC Psychiatry, 12, 107. http://dx.doi.org/10.1186/1471-244X-12-107

Egner, T., \& Gruzelier, J. H. (2002). Ecological validity of neurofeedback: modulation of slow wave EEG enhances musical performance. Cognitive Neuroscience and Neuropsychology, 14(9), 1221-1224. http://dx.doi.org /10.1097/00001756-200307010-00006

Escolano, C., Navarro-Gil, M., Garcia-Campayo, J., Congedo, M., De Ridder, D., \& Minguez, J. (2014). A controlled study on the cognitive effect of alpha neurofeedback training in patients with major depressive disorder. Frontiers in Behavioral Neuroscience, $\quad 8, \quad 296$. http://dx.doi.org/10.3389 /fnbeh.2014.00296

Faridnia, M., Shojaei, M., \& Rahimi, A. (2012). The effect of neurofeedback training on the anxiety of elite female swimmers. Annals of Biological Research, 3(2), 1020-1028.

Gardner, F., \& Moore, Z. E. (2004). A mindfulness-acceptancecommitment-based approach to athletic performance enhancement: Theoretical considerations. Behavior Therapy, 35(4), 707-723. http://dx.doi.org/10.1016/S00057894(04)80016-9

Gardner, F. L., \& Moore, Z. E. (2006). Clinical sport psychology. Champaign, IL: Human Kinetics.

Gardner, F. L., \& Moore, Z. E. (2007). The psychology of enhancing human performance: The MindfulnessAcceptance-Commitment (MAC) approach. New York: Springer Publishing.

Gardner, F. L., \& Moore, Z. E. (2012). Mindfulness and acceptance models in sport psychology: A decade of basic and applied scientific advancements. Canadian Psychology, 53(4), 309-318. http://dx.doi.org/10.1037/a0030220

Gevensleben, H., Holl, B., Albrecht, B., Vogel, C., Schlamp, D., Kratz, O., ... Heinrich, H. (2009). Is neurofeedback an efficacious treatment for ADHD? A randomised controlled clinical trial. Journal of Child Psychology and Psychiatry, 50(7), $\quad$ 780-789. http://dx.doi.org/10.1111/j.14697610.2008.02033.x

Goldberg, S. B., Del Re, A. C., Hoyt, W. T., \& Davis, J. M. (2014). The secret ingredient in mindfulness interventions? A case for practice quality over quantity. Journal of Counseling Psychology, 61(3), 491-497. http://dx.doi.org/10.1037 /cou0000032

Goodman, F. R., Kashdan, T. B., Mallard, T. T., \& Schumann, M. (2014). A brief mindfulness and yoga intervention with an entire NCAA Division I athletic team: An initial investigation. Psychology of Consciousness: Theory, Research, and Practice, 1(4), 339-356. http://dx.doi.org/10.1037 Icns0000022

Hammer, B. U., Colbert, A. P., Brown, K. A., \& Ilioi, E. C. (2011). Neurofeedback for insomnia: A pilot study of Z-score SMR and individualized protocols. Applied Psychophysiology and 
Biofeedback, 36, 251-264. http://dx.doi.org/10.1007/s10484011-9165-y

Hammond, D. C. (2007). Neurofeedback for the enhancement of athletic performance and physical balance. The Journal of the American Board of Sport Psychology, 1, 1-9.

Hammond, D. C. (2011). What is neurofeedback: an update. Journal of Neurotherapy, 15(4), 305-336. http://dx.doi.org/10.1080/10874208.2011.623090

Hanslmayr, S., Sauseng, P., Doppelmayr, M., Schabus, M., \& Klimesch, W. (2005). Increasing individual upper alpha power by neurofeedback improves cognitive performance in human subjects. Applied Psychophysiology and Biofeedback, 30(1), 1-10. http://dx.doi.org/10.1007/s10484-005-2169-8

Hindman, R. K., Glass, C. R., Arnkoff, D. B., \& Maron, D. D. (2014). A comparison of formal and informal mindfulness programs for stress reduction in university students. Mindfulness, 6(4), 873-884. http://dx.doi.org/10.1007/s12671014-0331-1

Janelle, C. M., \& Hatfield, B. D. (2008). Visual Attention and Brain Processes that Underlie Expert Performance: Implications for Sport and Military Psychology. Military Psychology, 20(Suppl. 1), S39-S69. http://dx.doi.org/10.1080/08995600701804798

Julian, L. J. (2011). Measures of anxiety: State-Trait Anxiety Inventory (STAI), Beck Anxiety Inventory (BAI), and Hospital Anxiety and Depression Scale-Anxiety (HADS-A). Arthritis Care and Research, 63(S11), S467-S472. http://dx.doi.org/10.1002/acr.20561

Kabat-Zinn, J. (1990). Full catastrophe living: Using the wisdom of your body and mind to face stress, pain, and illness. New York: Delacorte.

Kabat-Zinn, J. (1994). Wherever you go, there you are: Mindfulness meditation in everyday life. New York: Hyperion.

Kayiran, S., Dursun, E., Dursun, N., Ermutlu, N., \& Karamürsel, S. (2010). Neurofeedback intervention in fibromyalgia syndrome; a randomized, controlled, rater blind clinical trial. Applied Psychophysiology and Biofeedback, 35(4), 293-302. http://dx.doi.org/10.1007/s10484-010-9135-9

Kerr, C. E., Jones, S. R., Wan, Q., Pritchett, D. L., Wasserman, R. H., Wexler, A., ... Moore, C. I. (2011). Effects of mindfulness meditation training on anticipatory alpha modulation in primary somatosensory cortex. Brain Research Bulletin, 85(3-4), $\quad$ 96-103. http://dx.doi.org/10.1016 /j.brainresbull.2011.03.026

Kerr, C. E., Sacchet, M. D., Lazar, S. W., Moore, C. I., \& Jones, S. R. (2013). Mindfulness starts with the body: somatosensory attention and top-down modulation of cortical alpha rhythms in mindfulness meditation. Frontiers in Human Neuroscience, $\quad 7,12 . \quad$ http://dx.doi.org/10.3389 /fnhum.2013.00012

Lazar, S. W., Kerr, C. E., Wasserman, R. H., Gray, J. R., Greve, D. N., Treadway, M. T., ... Fischl, B. (2005). Meditation experience is associated with increased cortical thickness. Neuroreport, 16(17), 1893-1897. http://dx.doi.org/10.1097 101.wnr.0000186598.66243.19

Lofthouse, N., Arnold, L. E., Hersch, S., Hurt, E., \& DeBeus, R. (2012). A review of neurofeedback treatment for pediatric ADHD. Journal of Attention Disorders, 16(5), 351-372. http://dx.doi.org/10.1177/1087054711427530

Marchand, W. R. (2014). Neural mechanisms of mindfulness and meditation: Evidence from neuroimaging studies. World Journal of Radiology, 6(7), 471-479. http://dx.doi.org/10.4329/wjr.v6.i7.471

Marks, D. R. (2008). The Buddha's extra scoop: neural correlates of mindfulness and clinical sport psychology. Journal of Clinical Sport Psychology, 2(3), 216-241.

McDermott, K. B., \& Miller, G. E. (2007). Designing studies to avoid confounds. In R. J. Sternberg, H. L. Roediger III, \& D. F. Halpern (Eds.), Critical Thinking in Psychology (pp. 131142). New York, NY: Cambridge University Press.
Miller, M. W., Groman, L. J., Rietschel, J. C., McDonald, C. G., Iso-Ahola, S. E., \& Hatfield, B. D. (2013). The effects of team environment on attentional resource allocation and cognitive workload. Sport, Exercise, and Performance Psychology, 2(2), 77-89. http://dx.doi.org/10.1037/a0030586

Nichols, D. S. (1997). Balance retraining after stroke using force platform biofeedback. Physical Therapy, 77(5), 553-558.

Nyklíček, I., \& Kuijpers, K. F. (2008). Effects of mindfulness-based stress reduction intervention on psychological well-being and quality of life: Is increased mindfulness indeed the mechanism? Annals of Behavioral Medicine, 35(3), 331-340. http://dx.doi.org/10.1007/s12160-008-9030-2

Reinecke, K., Cordes, M., Lerch, C., Koutsandréaou, F., Schubert, M., Weiss, M., \& Baumeister, J. (2011). From lab to field conditions: a pilot study on EEG methodology in applied sports sciences. Applied Psychophysiology and Biofeedback, 36(4), 265-271. http://dx.doi.org/10.1007/s10484-011-9166-x

Shapiro, S. L., Carlson, L. E., Astin, J. A., \& Freedman, B. (2006). Mechanisms of mindfulness. Journal of Clinical Psychology, 62(3), 373-386. http://dx.doi.org/10.1002/jclp.20237

Sherlin, L. H., Arns, M., Lubar, J., Heinrich, H., Kerson, C., Strehl, U., \& Sterman, M. B. (2011). Neurofeedback and basic learning theory: Implications for research and practice. Journal of Neurotherapy, 15(4), 292-304. http://dx.doi.org/10.1080/10874208.2011.623089

Sherlin, L. H., Larson, N. C., \& Sherlin, R. M. (2013). Developing a Performance Brain Training Approach for Baseball: A Process Analysis with Descriptive Data. Applied Psychophysiology and Biofeedback, 38(1), 29-44. http://dx.doi.org/10.1007/s10484-012-9205-2

Smith, R. E., Schutz, R. W., Smoll, F. L., \& Ptacek, J. T. (1995). Development and validation of a multidimensional measure of sport-specific psychological skills: The Athletic Coping Skills Inventory-28. Journal of Sport and Exercise Psychology, 17, 379-398.

Spielberger, C. D., Gorsuch, R. L., Lushene, R., Vagg, P. R., \& Jacobs, G. A. (1983). Manual for the State-Trait Anxiety Inventory. Palo Alto, CA: Consulting Psychologists Press.

Stanley, E. A. (2014). Mindfulness-based mind fitness training (MMFT): An approach for enhancing performance and building resilience in high stress contexts. In A. Le, C. T. Ngnoumen, \& E. J. Langer (Eds.), The Wiley-Blackwell Handbook of Mindfulness, Volume II (pp. 964-985). West Sussex, UK: John Wiley \& Sons, Ltd.

Van der Hiele, K., Vein, A. A., Reijntjes, R. H. A. M., Westendorp, R. G. J., Bollen, E. L. E. M., van Buchem, M. A., ... Middelkoop, H. A. M. (2007). EEG correlates in the spectrum of cognitive decline. Clinical Neurophysiology, 118(9), 19311939. http://dx.doi.org/10.1016/j.clinph.2007.05.070

Van Erp, J. B. F., Reschke, S., Grootjen, M., \& Brouwer, A.-M. (2009). Brain performance enhancement for military operators. RTO-MP-HFM, 181(32), 1-16.

Walker, J. E. (2010). Recent advances in quantitative EEG as an aid to diagnosis and as a guide to neurofeedback training for cortical hypofunctions, hyperfunctions, disconnections, and hyperconnections: Improving efficacy in complicated neurological and psychological disorders. Applied Psychophysiology and Biofeedback, 35(1), 25-27. http://dx.doi.org/10.1007/s10484-009-9107-0

Williams, J., \& Bentman, S. (2014). An investigation into the reliability and variability of wobble board performance in a healthy population using the SMARTwobble instrumented wobble board. Physical Therapy in Sport, 15(3), 143-147. http://dx.doi.org/10.1016/j.ptsp.2013.08.003

Zeidan, F., Johnson, S. K., Diamond, B. J., David, Z., \& Goolkasian, P. (2010). Mindfulness meditation improves cognition: Evidence of brief mental training. Consciousness and Cognition, 19(2), 597-605. http://dx.doi.org/10.1016 /j.concog.2010.03.014 
Zoefel, B., Huster, R. J., \& Herrmann, C. S. (2011). Neurofeedback training of the upper alpha frequency band in EEG improves cognitive performance. Neurolmage, 54(2), $1427-1431$

http://dx.doi.org/10.1016/j.neuroimage.2010.08.078
Received: November 8, 2015

Accepted: November 8, 2015

Published: December 9, 2015 